\title{
¿Qué factores aumentan la aprobación al gobierno en un proyecto de infraestructura urbana? La construcción de la Línea 3 del Tren Ligero en el Área Metropolitana de Guadalajara, México
}

\author{
Which factors improve public acceptance of government in an \\ urban infrastructure project? Building the Line 3 of the Light Rail \\ in Guadalajara Metropolitan Area, Mexico
}

Fernando Calonge Reillo

Universidad de Guadalajara (México) ORCID: http://orcid.org/0000-0003-1788-2954

fercalonge@yahoo.es

\section{NOTA BIOGRÁFICA}

Fernando Calonge Reillo es Doctor en Sociología por la Universidad Complutense de Madrid. En la actualidad es profesor investigador de la Universidad de Guadalajara, México, e integrante del Sistema Nacional de Investigadores (CONACyT México) en su nivel I. Sus líneas de investigación son las dimensiones sociopolíticas de las infraestructuras de transporte.

\section{RESUMEN}

El papel de los grandes proyectos de infraestructuras urbanas es cada vez más importante a escala global y en la región latinoamericana. Con la implementación de esquemas de gobernanza urbana, es fundamental conocer cómo la ciudadanía evalúa la actuación gubernamental en este tipo de inversiones. Este artículo se basa en los resultados de una encuesta aleatoria desarrollada en el Área Metropolitana de Guadalajara, México. Sus principales resultados subrayan cómo la mejora de los mecanismos participativos y la reducción de los impactos directos a los vecinos, son las dimensiones más decisivas que coadyuvan a la aprobación de la actuación gubernamental.

\section{PALABRAS CLAVE}

Megaproyectos; Opinión ciudadana; Políticas de transporte; Transporte masivo.

\begin{abstract}
The role of megaprojects is increasingly outstanding both in a global scope and in the Latin-American region. With the deepening of urban governance, it is crucial to consider how citizens asset government performance in this kind of investments. This paper analyzes the results of a random survey conducted in Guadalajara Metropolitan Area, Mexico. The main results point that the enhancement of public participation and the compensation of the building impacts are the two most important factors in determining citizens' approval of government action.
\end{abstract}

\section{KEYWORDS}

Citizenship assessment; Mass Transit; Megaprojects; Transportation Policy. 
GAPP. Nueva Época - N. ${ }^{2}$ 1, Mayo-Octubre 2019 - ISSN: 1989-8991 - DOI: 10.24965/gapp.v0i21.10537 - [Págs. 109-124] ¿Qué factores aumentan la aprobación al gobierno en un proyecto de infraestructura urbana? La construcción de la Línea 3 del Tren..

Fernando Calonge Reillo

\begin{abstract}
SUMARIO
1. INTRODUCCIÓN. 2. LA GOBERNANZA URBANA Y LOS CONDICIONANTES DE LA OPINIÓN CIUDADANA. 3. CONTEXTO DE LA INVESTIGACIÓN Y METODOLOGÍA. 4. RESULTADOS. 4.1. EXPOSICIÓN A LOS IMPACTOS DE LA OBRA. 4.2. LA OPINIÓN GENERAL SOBRE LA OBRA. 4.3. LA OPINIÓN SOBRE LA INFLUENCIA DE LA OBRA EN LA COLONIA. 4.4. LA PARTICIPACIÓN EN LA DETERMINACIÓN DE LAS INFRAESTRUCTURAS URBANAS. 5. CONCLUSIONES.
\end{abstract}

\title{
1. INTRODUCCIÓN
}

Las inversiones en infraestructuras urbanas desempeñan un papel preponderante en los destinos de las metrópolis actuales. Distintos factores influyen en esa importancia: la creciente competencia en una escala global que se plantea entre las principales ciudades; la pretensión de dinamizar las economías urbanas; la respuesta a los crecimientos poblacionales acelerados que se dan en las ciudades de los países en desarrollo, etc. Con estas inversiones, al cabo de pocos años, se producen transformaciones de gran calado que alteran las dinámicas de sectores muy amplios de la metrópolis (Flyvbjerg, 2014: 6).

La importancia creciente de las formas de administración basadas en la gobernanza, con la descentralización del poder y su reparto a lo largo de distintos sectores de la ciudadanía (Dávila y Brand, 2012: 90), impone otros retos al papel de los gobiernos en la planificación, construcción y administración de este tipo de infraestructuras. En especial, se hace cada vez más necesario reparar en cómo estos proyectos se socializan y son recibidos por parte de la ciudadanía.

Este artículo analiza algunos condicionantes que influyen en la forma como los ciudadanos evalúan el papel de los distintos niveles de gobierno en la planificación y construcción de estas infraestructuras. En especial aborda cómo la ciudadanía aprecia la actuación gubernamental en el caso concreto de la construcción de la Línea 3 del Tren Ligero en el Área Metropolitana de Guadalajara, México (L3TL). El propósito es contribuir al debate existente sobre los condicionantes de la opinión ciudadana a la hora de evaluar este tipo de grandes proyectos urbanos. En particular, los resultados destacan la importancia que tiene mejorar los procesos participativos previos, y el cuidado en minimizar los impactos durante la construcción, para garantizar un mayor apoyo en el desarrollo de esta infraestructura.

Para hacerlo se sitúan teóricamente las actuales formas de gestión basadas en la gobernanza urbana, enfatizándose el papel que desempeñan los procesos participativos. Dentro de esta tendencia general, se discutirá específicamente el desarrollo de los procesos de comunicación y recepción de discursos alrededor de la construcción de infraestructuras, y se repasarán algunos condicionantes que se han señalado que inciden en la contingente valoración pública de este tipo de inversiones. En un segundo momento se presentan los principales resultados de la encuesta, organizados en cuatro dimensiones que condicionan la evaluación ciudadana sobre el papel de los gobiernos en la implementación de infraestructuras: la exposición a los impactos negativos derivados de la construcción de la obra, la percepción ciudadana sobre cómo la obra altera y alterará las dinámicas locales en el entorno, la valoración general de la ciudadanía sobre la conveniencia de la obra, y el grado como se involucra y participa en su gestión. Las conclusiones finales mostrarán la importancia que se debe dar a los procesos participativos, y la necesidad de aminorar y compensar los impactos directos que se sufren como los dos medios que mejor explican la aprobación de la ciudadanía sobre la actuación gubernamental en la implementación de grandes infraestructuras.

\section{LA GOBERNANZA URBANA Y LOS CONDICIONANTES DE LA OPINIÓN CIUDADANA}

La construcción de grandes proyectos de infraestructura urbana es objeto de la atención pública por la dimensión de las consecuencias que acarrea para la vida de los habitantes de las metrópolis. Dada la polémica que suele rodear este tipo de obras, se insiste en la necesidad de cuidar su gestión y en general la forma como se insertan dentro de la más general gobernanza urbana (Flyvbjerg, 2009: 353).

Los esquemas de gobernanza son claves para entender la relación entre las instituciones, la ciudadanía, y la implementación de proyectos de intervención urbana. En términos más generales, la gobernanza ha significado abandonar tradicionales procesos verticales de toma de decisiones, para apostar por afrontar la gestión desde la interdependencia entre distintos tomadores de decisiones en procesos más horizontales y que intentan incluir a amplios espectros de la ciudadanía (Sack, 2011: 47; Bakker, 2010: 45). Esta preten- 


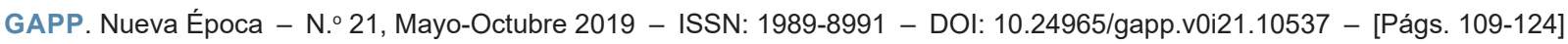

¿Qué factores aumentan la aprobación al gobierno en un proyecto de infraestructura urbana? La construcción de la Línea 3 del Tren..

Fernando Calonge Reillo

sión de romper esquemas verticales ha acarreado acercar la toma decisiones al ámbito local y comunitario (Manzi et al., 2010: 19), y ha concluido con la instauración de fórmulas institucionalizadas de gobernanza urbana (Stren y Polese, 2000: 17).

La participación ciudadana adquiere una gran importancia en la puesta en marcha de estos procesos de gobernanza urbana porque garantiza una amplia implicación de un variado número de actores en la toma de decisiones. Mejorar los procedimientos de participación incide en el éxito y en la apropiación de las distintas políticas e inversiones (Allen y Lloyd Jones, 2010; 68), y en la formación de las actitudes de los ciudadanos respecto a sus gobiernos y sus iniciativas (Jordhus-Lier, 2015: 172; Kash e Hidalgo, 2014). De ahí que se insista en la necesidad de institucionalizar estos procesos de participación, para evitar que queden como puntuales episodios de consulta (McCright y Clark, 2006: 203)

Sin embargo, se han constatado serias dificultades para la materialización de la gobernanza que alentarían los recelos y reparos sobre las inversiones en infraestructura urbana. En primer lugar se indica que proyectos de gran envergadura como el que es objeto de análisis en este artículo, suelen entenderse desde las urgencias de perder las inversiones prometidas lo que propende a generar procesos poco claros y abiertos (Smith, 2010: 206; Flyvbjerg, 2005: 20). Asimismo, se insiste en las dificultades por canalizar adecuadamente todos los intereses ciudadanos, y no sólo los socialmente hegemónicos (Manzi et al., 2010: 22; Bon, 2015: 228; Norton, 2002: 136).

Existen dificultades específicas a la implantación de la gobernanza urbana dentro de la región latinoamericana. En este contexto, es especialmente fuerte la tradición tecnocrática y autoritaria en la planificación de las infraestructuras (Keck, 2002: 170; da Silva, 2010: 220; Moreno Carranco, 2013: 187) lo que impide la apertura de los procesos participativos. En específico, la persistencia de estas formas de entender la relación entre gobernantes y gobernados, acarrea frecuentes intentos por cooptar determinadas capas de población, especialmente las más vulnerables o deja las experiencias participativas en llanas campañas informativas (Valenzuela, 2013: 108), Además, hay que consignar la gran debilidad institucional que impide que se formalicen y rutinicen los procesos participativos (Bakker, 2010: 29) y que la participación quede instalada definitivamente en la agenda urbana.

La comprensión y evaluación que realizan los ciudadanos de la actuación gubernamental en la implementación de infraestructura urbana es muy importante para una efectiva implantación de la gobernanza urbana. Desde una postura crítica, pero algo simplificadora, la literatura ha enfatizado el sentido ideológico que acarrea buena parte de las estrategias de comunicación gubernamentales y del propio despliegue de las infraestructuras. Especialmente relevante para el contexto de un país en desarrollo, se ha subrayado cómo el intento por dominar a poblaciones y territorios «atrasados» y marginados se ha realizado a través ciertos relatos sobre la modernización y el progreso (Kaika y Swyngedouw, 2000: 125; Siemiatycki, 2005: 30). La infraestructura, en su papel de elemento modernizador (Schot, 2003: 257), habría sido un arma ideológica muy importante (Dupont, 2011: 544), en la medida en que prometía mejorar los estándares de vida y las libertades (Larkin, 2013: 332), en que conseguía acallar posibles voces disidentes (Siemiatycki, 2006: 287), o en que implementaba exhibiciones públicas de dominio y superioridad tecnológicas difíciles de resistir (Anand, 2011: 547; Larkin, 2008: 39).

Aunque algunos de estos estudios asumen la naturaleza contingente y abierta a disputa de esa comunicación de significados y experiencias a través de la infraestructura (Harvey, 2012), prevalece un tipo de análisis que establece que la capacidad de los estados para desplegar determinadas infraestructuras es equivalente con su capacidad por incidir en el estado de opinión y en las evaluaciones de los ciudadanos. Frente a estos análisis, se propone aquí partir de la perspectiva crítica en teoría de la comunicación, que insiste en la necesidad de atender mucho más de cerca a los procesos de apropiación, decodificación y discusión de los mensajes por parte de los públicos receptores (Mattelart y Mattelart, 1997: 34-41). En nuestro caso, no se puede tomar la opinión ciudadana como un hecho que responde necesariamente a las formas infraestructurales establecidas o a las estrategias de comunicación de los estados. Al contrario, se propone prestar atención al sentido contingente de la formación de la opinión ciudadana (Valentin y Bougs, 2013: 563) como consecuencia de los variados procesos de apropiación e interpretación (Humphrey, 2003: 43; Bunnell, 2002: 1687). En particular, este artículo intentará explorar algunos factores y condicionantes que pueden incidir en esa variabilidad de la opinión ciudadana.

Así, se ha indicado que la atención selectiva que la ciudadanía presta a determinadas campañas gubernamentales (Janse y Konijnendijk, 2007: 35), que la intercesión de los intereses propios que pueden ser dañados por aquellos intereses supuestos que la obra pretende alentar (Ruming, 2014: 261), que la confianza depositada en el gobierno para aceptar o controvertir sus mensajes (Battaglia et al., 2014: 798; Marincioni y Appioti, 2009: 864), o que el fallo en los propios canales gubernamentales de transmisión de la información 


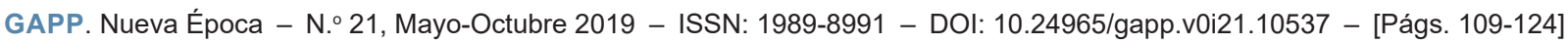

¿Qué factores aumentan la aprobación al gobierno en un proyecto de infraestructura urbana? La construcción de la Línea 3 del Tren..

Fernando Calonge Reillo

(Bon, 2015: 45), son otros tantos factores que inciden en la gran variabilidad como finalmente pueden ser descodificados los mensajes prendidos de la infraestructura urbana.

A la hora de revisar la formación de la opinión pública, pueden establecerse varios condicionantes que inciden en esta variabilidad. En los países latinoamericanos y en desarrollo es preciso contar con las formas habituales de prestación de la infraestructura urbana para conocer la predisposición que pueden guardar los habitantes hacia estas infraestructuras modernizadoras. El enfoque autoritario y tecnocrático que ya se ha comentado en la implementación de infraestructuras condiciona que buena parte de la ciudadanía albergue amplios recelos en los momentos en que los gobiernos impulsan los proyectos de modernización a través de la construcción de infraestructuras (Khan, 2006: 88). Esos recelos integran sospechas de que las nuevas infraestructuras van a favorecer a los inversores e intereses internacionales (Dalakoglou, 2010: 138; PagetSeekins, 2015: 118), de que las necesidades locales seguirán quedando insatisfechas (Turner, 2012: 51) o de que las formas de vida e identidades locales quedarán amenazadas (Pérez Negrete, 2016: 343; Novy y Peters, 2013: 250; Grove, 2009: 214).

La resistencia se agudiza en el instante en que el inicio de la construcción de la infraestructura abre una serie de incomodidades a las poblaciones que conviven con las obras. Así, las afectaciones al entorno construido próximo a los residentes (Barker, 2005: 710) o el deterioro ambiental que suele comportar la construcción de magaproyectos (Evans, 2002: 16), motivan la oposición de buena parte de la población frente a las actuaciones gubernamentales.

Los ciudadanos pueden valorar negativamente la acción de los gobiernos, si antes anticipan que su día a día en sus colonias y vecindarios va a verse negativamente alterado. Así puede suceder con las infraestructuras que ponen en marcha procesos de «gentrificación» que desplazan a las poblaciones pobres locales (Manzi, 2010: 44), que agudizan las brechas y las fracturas sociales y espaciales entre colonias adineradas y colonias marginadas (Levine, 2000: 134), o que suprimen comercios populares y a veces informales que son fundamentales para el abasto cotidiano de los hogares (Turner, 2012: 51). En cualquiera de estos casos, la evaluación de los cambios futuros que sobrevendrán con la construcción de la infraestructura es sentida como una pérdida de autonomía (Hess, 2009: 54) que pone en marcha procesos de oposición frente a las actuaciones del gobierno.

Por otro lado, los ciudadanos también pueden prever que la construcción de la infraestructura traiga consecuencias favorables para los vecindarios donde desarrollan su cotidianidad. Así ha sucedido con ciertas inversiones en infraestructuras de transporte masivo, que en muchos casos son aprobadas porque se prevé que mejorarán las conexiones y la inclusión del vecindario en el resto de la metrópolis (Grieco, 2015: 93; Brodzinsky, 2014: 78). Así, es habitual que, ante las noticias de la implantación de nueva infraestructura en sus entornos, los vecinos realicen procesos de evaluación en donde analizan minuciosamente los beneficios y perjuicios que puede tener la construcción de la infraestructura (Dietz y Atkinson, 2005: 446).

Entre las condiciones que se ha identificado como claves para obtener mejores niveles de valoración ciudadana está la apertura de procesos participativos, como quedaban implicados en los nuevos esquemas de gobernanza urbana. Así, si los procesos son verdaderamente participativos, y se extienden a aspectos de la formación de las políticas de infraestructuras (Keck, 2002: 184; Schneier-Madanes, 2005: 165), pueden acarrear un mayor apoyo a las actuaciones gubernamentales, y esto por varias razones. En primer lugar la participación ciudadana puede garantizar futuros abastos de los servicios urbanos a las poblaciones especialmente más vulnerables, con lo que se puede lograr así una mayor aceptación de la infraestructura (Evans, 2002: 15). En segundo lugar, la mejora de la participación ciudadana suele darse vis a vis con un fortalecimiento de las administraciones locales (Marincioni y Appioti, 2009: 864), lo que refuerza el sentido de cercanía y utilidad de la obra. Finalmente, la participación ciudadana reiterada mejora los procesos de retroalimentación de las administraciones y también de los cuerpos de técnicos con la ciudadanía, lo que garantiza poder introducir puntualmente mejoras en la planificación y construcción que sean consideradas de utilidad por parte de la población (Schot, 2003: 274; Battaglia, et al., 2014: 797).

En definitiva, los nuevos esquemas de gobernanza urbana implican nuevas formas de afrontar la gestión de las infraestructuras que pueden ser determinantes en la formación de las opiniones de la ciudadanía. El caso latinoamericano, con su dilatada historia de gestión tecnocrática y autoritaria de la implantación de la infraestructura, impone una particularidad que será abordada en este artículo. Contando con las dificultades por implantar en la región la gobernanza urbana, y con los recelos y suspicacias de parte de la ciudadanía ya documentados, se mostrará cómo las mejoras en los procesos de participación, y una atención más cuidadosa hacia las afectaciones que puede implicar para los vecinos son cruciales para explicar variaciones en la evaluación ciudadana de las infraestructuras. 


\section{CONTEXTO DE LA INVESTIGACIÓN Y METODOLOGÍA}

La L3TL comenzó a construirse en agosto de 2014, y se espera que entre en operación a finales de 2018. Como se observa en el Mapa 1, esta línea complementa la línea 1 del tren ligero, que se terminó de construir en 1989, la línea 2, que entró en operaciones en 1994, y la única línea de BRT existente en la ciudad, el Macrobús, que se inauguró en 2009, como medios de transporte masivo. Su trazado discurrirá en la diagonal noroeste-sureste a lo largo de 21,5 kilómetros, atravesando colonias de muy diferentes características en términos habitacionales y socio-demográficos.

\section{MAPA 1. ESQUEMA DEL TRANSPORTE MASIVO EN EL ÁREA METROPOLITANA DE GUADALAJARA}

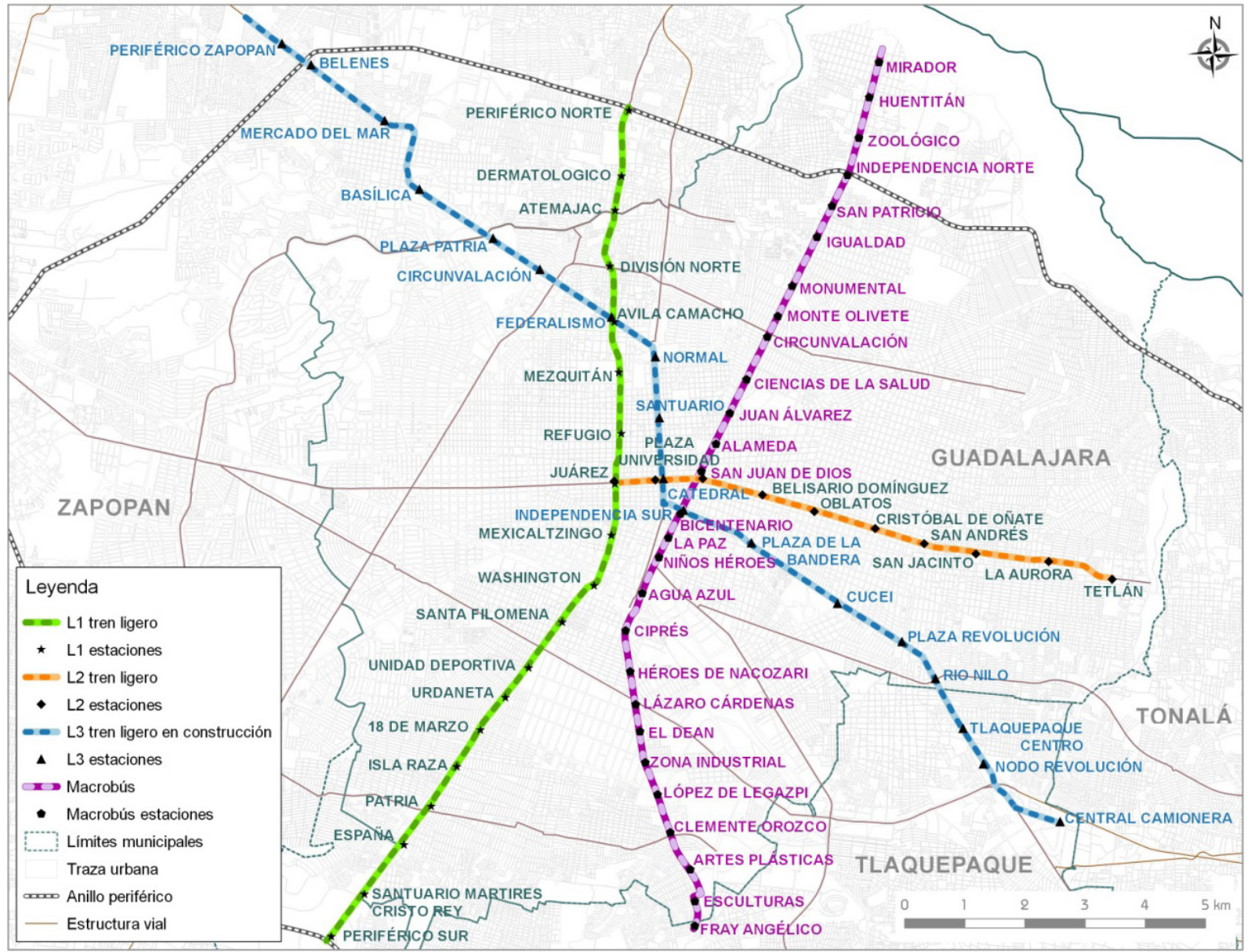

Fuente: elaboración propia.

La ampliación del transporte masivo en la metrópolis fue muy conflictiva y altamente politizada. Los diferentes partidos políticos proponían medios de transporte y derroteros diferentes, pero con la llegada al poder del Partido Revolucionario Institucional en 2014, tanto a nivel federal como a nivel estatal, se optó por recurrir a la solución más costosa: una tercera línea del tren ligero que discurriera por la referida diagonal. Su presupuesto inicial fue de 0,8 mil millones de euros; sin embargo este montante se ha ido incrementando con el transcurrir de los meses, de manera que pasó a 0,9 mil millones de euros en diciembre de 2015; en septiembre de 2016 la desviación ya alcanzaba los mil millones de euros y en abril de 2018 se hablaba de 1,15 mil millones de euros. Según el estudio de demanda, se prevé que con una reestructuración intensa de rutas de autobuses urbanos en las inmediaciones, la línea pueda alcanzar a captar 164.916 ascensos diarios. Para contextualizar estos datos, hay que señalar que, por ejemplo, el sistema de Macrobús existente, que discurre por la Calzada Independencia y rutas alimentadoras, a lo largo de 16 kilómetros, tuvo un costo de 38 millones de euros, y transportó en 2015 un promedio de 119.150 pasajeros diarios.

La L3TL y el Área Metropolitana de Guadalajara no son ajenos a lo que sucede en la región latinoamericana. Con la excepción de casos aislados de buenas prácticas, como Curitiba, Medellín o Bogotá, los re- 
zagos en la construcción de infraestructura urbana se acumulan por decenios y la respuesta gubernamental suele ser titubeante. En particular se constata la realización de inversiones que han beneficiado al automóvil y un olvido reincidente del transporte masivo que sólo en la actualidad se empieza a enmendar. Desde una larga tradición de tecnocracia, autoritarismo y opacidad, las agencias gubernamentales aún no disponen de los mecanismos y las inercias suficientes para el impulso a la infraestructura urbana desde los nuevos esquemas de la gobernanza urbana. La L3TL es a este respecto representativa de la región, por todos los retos que implica para la construcción de infraestructuras de transporte masivo el enfrentar decididamente una mayor participación y aceptación de la ciudadanía afectada.

Los resultados que aquí se presentan provienen de una encuesta que se desarrolló gracias al apoyo de la Convocatoria de Atención a Problemas Nacionales de CONACyT, en el año 2014. La encuesta consta de 800 elementos muestrales aplicados en mayo de 2016 dentro de una franja de 1 kilómetro de distancia respecto al derrotero del tren. Ese tamaño muestral permite establecer generalizaciones al conjunto de la población con un margen de error de $+/-3,46 \%$, para un nivel de confianza del $95 \%$.

Como variables dependientes que sirven para medir la opinión de los ciudadanos sobre la actuación del gobierno, se construyó una escala, que en el cuestionario quedó integrada bajo la siguiente pregunta: De 0 a 10, siendo 0 nada adecuado, y 10 totalmente adecuado, ¿cómo calificaría las siguientes actuaciones relacionadas con la construcción de la L3TL? Esta pregunta se aplicó para los siguientes aspectos:

- Información sobre las características de la obra.

- Información sobre el cierre de vialidades.

- Información de impactos ambientales en su colonia.

- Información sobre apoyos del gobierno a los afectados.

- Posibilidad de presentar quejas y denuncias sobre las actividades de la obra.

Para realizar un primer acercamiento a los factores que inciden en los distintos niveles de aprobación de la construcción se ha realizado una comparación de medias de nuestra variable dependiente (calificación de 0 a 10 de las actuaciones gubernamentales) según los valores categóricos de una serie de variables dependientes que más adelante se enumeran. Para comprobar la significatividad de las diferencias de medias se aplicó el estadístico T de Student.

Para profundizar en el análisis de los factores que inciden en la aprobación ciudadana de los distintos componentes de la construcción de la obra se procedió a aplicar diversos modelos de regresión logística binaria. La regresión logística binaria se pregunta por cómo las diferentes variables independientes aumentan o disminuyen la probabilidad de que una variable dependiente dicotomizada tome uno u otro valor. En nuestro caso, la variable dependiente de la calificación de las actuaciones relacionadas con la construcción de la L3TL ha sido dicotomizada en aprobado y suspenso; todas aquellas calificaciones que en nuestra escala quedaban 5 y 10 han sido transformadas en aprobado, y el resto en suspenso. Así, la aplicación de la regresión logística binaria para nuestro caso de estudio nos ha permitido identificar cómo los distintos factores aumentaban o disminuían la probabilidad de que los ciudadanos aprobaran la construcción de la referida infraestructura.

Para la identificación de los posibles factores, se ha retomado parte de la indagación teórica realizada que permitía identificar condiciones que influían en la evaluación ciudadana de la construcción de infraestructuras. Estos factores se trasladaron al cuestionario en la forma de las siguientes preguntas:

1. Exposición a los impactos de la obra. Variables:

- Colindar o no colindar con la obra.

- Percibir impactos durante la construcción en lo relativo a: Vertidos de combustibles, malos olores, vibraciones por obras y maquinaria y presencia basuras y residuos.

2. Opinión general sobre la obra. Preguntas:

- ¿Es necesaria la construcción de la L3TL?

- ¿Quiénes son los mayores beneficiados de la construcción de la L3TL?

3. Índice sobre la influencia de la obra en la colonia en el presente y en el futuro. Construido con los siguientes aspectos y dimensiones de valoración:

- La seguridad ciudadana.

- La existencia de parques y áreas verdes.

- La facilidad para caminar por la colonia. 
GAPP. Nueva Época - N. ${ }^{\circ}$ 21, Mayo-Octubre 2019 - ISSN: 1989-8991 - DOI: 10.24965/gapp.v0i21.10537 - [Págs. 109-124]

¿Qué factores aumentan la aprobación al gobierno en un proyecto de infraestructura urbana? La construcción de la Línea 3 del Tren..

- La limpieza e imagen de la colonia.

- La presencia de vecinos en las calles y la animación del barrio.

4. Participación en la determinación de infraestructuras urbanas. Variable:

- Participar en reuniones o asambleas de vecinos relacionadas con la construcción.

\section{RESULTADOS}

Las disputas que han rodeado la construcción de la L3TL todavía continúan, y se trasladan en una pobre valoración de la actuación del gobierno en los diferentes aspectos aquí sometidos a examen, según se aprecia en la Tabla 1.

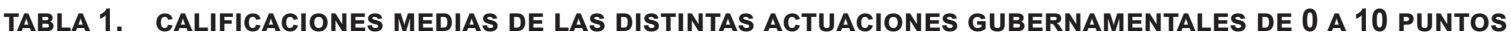

\begin{tabular}{lc}
\hline \multicolumn{1}{c}{ Aspecto de actuación gubernamental } & Calificación media \\
\hline Información características de la obra & 5,03 \\
\hline Información cierres vialidades & 5,01 \\
\hline Información impactos ambientales en colonia & 4,09 \\
\hline Información apoyos a afectados & 3,03 \\
\hline Posibilidad presentar quejas & 2,91 \\
\hline
\end{tabular}

Fuente: elaboración propia.

Las actuaciones que comportan informaciones más genéricas (sobre las características de la obra y los cierres y desvíos en las vialidades) son las que obtienen mejor calificación. Con el resto de aspectos más controvertidos y que se dejan sentir más sobre el día a día de los ciudadanos, las calificaciones bajan considerablemente.

A continuación se analizará con mayor detenimiento qué dimensiones se encuentran detrás de las diferentes valoraciones de la actuación del gobierno. Para ello se realizó una comparación de medias, aplicando la prueba $T$ de Student para establecer la significatividad de los resultados, y se realizaron modelos de regresión logística binaria para intentar comprobar cómo determinadas variables incidían en las opciones de aprobar la gestión gubernamental.

\subsection{Exposición a los impactos de la obra}

Uno de los factores que debería incidir en una mayor exposición a los impactos de la obra es el ubicarse en la proximidad de su construcción. Sin embargo los resultados obtenidos no indican que este factor sea decisivo para explicar la valoración diferencial sobre la actuación gubernamental. La cercanía a la obra sólo induce diferencias de medias significativas a nivel estadístico en la valoración sobre la información de las características de la obra, y sobre la información de impactos ambientales en la obra, según se aprecia en la Tabla 2.

TABLA 2. DIFERENCIAS DE MEDIAS SIGNIFICATIVAS EN LAS CALIFICACIONES DE LOS CIUDADANOS A LA ACTUACIÓN GUBERNAMENTAL, SEGÚN DISTANCIA AL TRAZADO

\begin{tabular}{lccc}
\hline $\begin{array}{l}\text { Aspectos de la actuación } \\
\text { gubernamental evaluados } \\
\text { por la ciudadanía }\end{array}$ & $\begin{array}{c}\text { Valoración por los } \\
\text { ciudadanos que colindan } \\
\text { con la infraestructura }\end{array}$ & $\begin{array}{c}\text { Valoración por los } \\
\text { ciudadanos que no colindan } \\
\text { con la infraestructura }\end{array}$ & \begin{tabular}{c} 
Sig. \\
\hline Información características de la obra
\end{tabular} \\
\hline Información impactos ambientales colonia & 6,04 & 4,92 & 0,003 \\
\hline
\end{tabular}

Fuente: elaboración propia. 
Puesto que el plan del gobierno consistía en realizar campañas informativas en un radio de 500 metros respecto al trazado, se comprueba que existen mejores valoraciones sobre cómo se informó de las características de la obra, y sobre los impactos ambientales que existirían en la colonia en aquellos domicilios que colindan con la construcción de la infraestructura y donde sí se tuvo la opción de acudir a dichas campañas.

Las variables que recogen los impactos percibidos en la colonia tienen mucha más influencia en cómo los ciudadanos valoran la actuación del gobierno. La Tabla 3 muestra esa valoración de la actuación del gobierno, para los impactos percibidos que han mostrado tener una mayor influencia en esa misma valoración.

TABLA 3. DIFERENCIAS DE MEDIAS EN LA CALIFICACIÓN DE LA ACTUACIÓN GUBERNAMENTAL, SEGÚN EL TIPO DE IMPACTO PERCIBIDO EN LA COLONIA QUE INFLUYE MÁS EN DICHA CALIFICACIÓN

\begin{tabular}{|c|c|c|c|c|}
\hline $\begin{array}{l}\text { Aspectos de la actuación } \\
\text { gubernamental evaluados } \\
\text { por la ciudadanía }\end{array}$ & $\begin{array}{l}\text { Tipo de impacto } \\
\text { percibido }\end{array}$ & $\begin{array}{l}\text { Valoración media } \\
\text { del aspecto de los } \\
\text { ciudadanos que } \\
\text { sí perciben ese } \\
\text { impacto }\end{array}$ & $\begin{array}{c}\text { Valoración media } \\
\text { del aspecto de los } \\
\text { ciudadanos que } \\
\text { no perciben ese } \\
\text { impacto }\end{array}$ & Sig. \\
\hline $\begin{array}{l}\text { Información características } \\
\text { de la obra }\end{array}$ & Vertidos de combustibles & 3,98 & 5,22 & 0,000 \\
\hline Información cierres vialidades & Vertidos de combustibles & 3,73 & 5,24 & 0,000 \\
\hline $\begin{array}{l}\text { Información impactos } \\
\text { ambientales en colonia }\end{array}$ & Malos olores & 2,52 & 4,33 & 0,000 \\
\hline $\begin{array}{l}\text { Información apoyos } \\
\text { a afectados }\end{array}$ & $\begin{array}{l}\text { Vibraciones por obras } \\
\text { y maquinaria }\end{array}$ & 2,23 & 3,41 & 0,000 \\
\hline Posibilidad presentar quejas & $\begin{array}{l}\text { Presencia basuras } \\
\text { y residuos }\end{array}$ & 2,05 & 3,19 & 0,000 \\
\hline
\end{tabular}

Fuente: elaboración propia.

El consignar presencia de vertidos de combustible es el impacto que mayor influencia tiene en cómo se califica de manera diferente la actuación del gobierno tanto en la información de las características de las obras, como en los cierres de las vialidades. No percibir ese impacto, en el primer caso implica otorgar una calificación un $31 \%$ superior, y en el segundo implica otorgar una calificación un $40 \%$ superior. Los malos olores son el impacto que más influye en que se califique de forma diferente la actuación del gobierno en materia de impactos ambientales (no percibirlos implica otorgar una calificación un $72 \%$ superior). El observar vibraciones derivadas de la obra es el impacto más determinante en la calificación que se da a la información sobre los apoyos existentes a los afectados (no observarlas comporta calificar esta actuación con un $53 \%$ más de nota). Finalmente, la presencia de basuras y residuos es el impacto más decisivo para evaluar de manera diferente las facilidades dadas en la presentación de quejas (no advertirlas en la colonia sube la calificación media en un $56 \%$ ).

Por último, se realizó una regresión logística binaria con el objetivo de calibrar de qué manera la percepción de los diferentes impactos aumentaba las opciones de aprobar la actuación del gobierno en sus distintas facetas. Todas las variables aquí analizadas fueron significativas y se integraron en los diferentes modelos explicativos. Los resultados obtenidos se expresan en la Tabla 4.

TABLA 4. INCREMENTO DE OPCIONES DE APROBAR LA ACTUACIÓN DEL GOBIERNO SEGÚN LA NO EXISTENCIA DE LOS IMPACTOS AMBIENTALES MÁS DETERMINANTES

\begin{tabular}{|c|c|c|c|}
\hline $\begin{array}{l}\text { Aspectos de la actuación } \\
\text { gubernamental }\end{array}$ & $\begin{array}{l}\text { Ausencia de percepción } \\
\text { de impacto }\end{array}$ & $\begin{array}{l}\text { Incremento de opciones } \\
\text { de aprobar la actuación }\end{array}$ & Sig. \\
\hline Información características de la obra & Vertidos de combustibles & 1,912 & 0,007 \\
\hline Información cierres vialidades & Vertidos de combustibles & 2,506 & 0,000 \\
\hline
\end{tabular}


GAPP. Nueva Época - N. ${ }^{\circ}$ 21, Mayo-Octubre 2019 - ISSN: 1989-8991 - DOI: 10.24965/gapp.v0i21.10537 - [Págs. 109-124] ¿Qué factores aumentan la aprobación al gobierno en un proyecto de infraestructura urbana? La construcción de la Línea 3 del Tren..

Fernando Calonge Reillo

\begin{tabular}{|c|c|c|c|}
\hline $\begin{array}{l}\text { Aspectos de la actuación } \\
\text { gubernamental }\end{array}$ & $\begin{array}{l}\text { Ausencia de percepción } \\
\text { de impacto }\end{array}$ & $\begin{array}{l}\text { Incremento de opciones } \\
\text { de aprobar la actuación }\end{array}$ & Sig. \\
\hline $\begin{array}{l}\text { Información impactos ambientales } \\
\text { en colonia }\end{array}$ & Malos olores & 2,830 & 0,000 \\
\hline Información apoyos a afectados & Vibraciones por obras y maquinaria & 1,996 & 0,000 \\
\hline Posibilidad presentar quejas & Presencia basuras y residuos & 2,058 & 0,000 \\
\hline
\end{tabular}

Fuente: elaboración propia.

El impacto que mayor influencia ha tenido sobre la calificación de la actuación del gobierno en los distintos aspectos ha sido el advertir malos olores, de manera que no percibirlos comporta multiplicar por 2,830 las opciones de aprobar la actuación del gobierno en materia de información sobre los impactos ambientales en la colonia. También importante ha sido la influencia de la percepción de vertidos de combustibles sobre la calificación de la actuación del gobierno en los cierres de vialidades (no percibir vertidos multiplica por 2,506 las opciones de aprobar la actuación del gobierno en esta materia).

\subsection{La opinión general sobre la obra}

En nuestro caso la opinión general sobre la obra se midió a través de dos variables, la que señala la opinión de la ciudadanía sobre la necesidad en sí de la infraestructura, y la que indica qué actores serán los más beneficiados por su implementación.

En el primer caso podemos hablar de que quienes piensan que la obra era necesaria, tienden a calificar mejor la actuación del gobierno en sus diferentes aspectos. Todas las diferencias de medias encontradas son estadísticamente significativas, según se observa en la Tabla 5.

TABLA 5. DIFERENCIAS de MEDIAS EN LAS CALIFICACIONES DE LOS CIUDADANOS A LA ACTUACIÓN GUBERNAMENTAL, SEGÚN OPINIÓN SOBRE LA NECESIDAD DE LA INFRAESTRUCTURA

\begin{tabular}{lccc}
\hline $\begin{array}{l}\text { Aspectos de la actuación } \\
\text { gubernamental evaluados } \\
\text { por la ciudadanía }\end{array}$ & $\begin{array}{c}\text { Valoración media que dan } \\
\text { quienes opinan que sí es } \\
\text { necesaria la obra }\end{array}$ & $\begin{array}{c}\text { Valoración media que dan } \\
\text { quienes opinan que no es } \\
\text { necesaria la obra }\end{array}$ & \begin{tabular}{c} 
Sig. \\
\hline Información características de la obra
\end{tabular} \\
\hline Información cierres vialidades & 5,34 & 4,52 & 0,000 \\
\hline Información impactos ambientales en colonia & 5,36 & 4,43 & 0,000 \\
\hline Información apoyos a afectados & 4,39 & 3,57 & 0,000 \\
\hline Posibilidad presentar quejas & 3,19 & 2,77 & 0,044 \\
\hline
\end{tabular}

Fuente: elaboración propia.

La diferencia más marcada se da en el caso de la valoración sobre la información de los impactos ambientales en la colonia. Aquí, quienes pensaban que la construcción sí era necesaria valoran un $23 \%$ mejor la actuación gubernamental que quienes pensaban que la obra no era necesaria. Otra diferencia de medias también resaltable es la que se da en el aspecto de la información de sobre los cierres de las vialidades. Pensar que la obra era necesaria conlleva calificar con un $21 \%$ más de nota la actuación del gobierno en este rubro.

Como se ve, las diferencias de medias en este caso son menores que en el caso anterior de la percepción sobre los impactos en la colonia, lo que motiva que en la mayor parte de los casos, esta variable sobre la necesidad de la obra no sea significativa a la hora de entrar en los modelos resultantes de la correlación logística binaria. Sólo ha entrado dentro del modelo la opinión sobre la necesidad de la L3TL en el caso de valorar la información sobre los cierres de vialidades. De esta forma, pensar que la L3TL era necesaria mul- 
tiplica por 1,735 las opciones de que los ciudadanos aprueben la actuación del gobierno a la hora de proveer información de los cierres de vialidades (sig. 0,002).

La consideración de los principales beneficiarios de la L3TL tiene una influencia modesta a la hora de evaluar la actuación de los distintos niveles de gobierno. Las diferencias más claras se presentaron para quienes respondían que el principal beneficiario iba a ser el gobierno, por lo que los valores de la variable se dicotomizaron entre quienes pensaban que iba a ser este actor el más beneficiado, y quienes optaron por el resto de posibilidades. Como muestra la Tabla 6 , todas las diferencias de medias resultaron ser significativas, excepto para el aspecto de la información sobre los impactos ambientales en la colonia.

TABLA 6. DIFERENCIAS DE MEDIAS EN LAS CALIFICACIONES DE LOS CIUDADANOS A LA ACTUACIÓN GUBERNAMENTAL, SEGÚN OPINIÓN DEL PRINCIPAL BENEFICIARIO DE LA OBRA

\begin{tabular}{lccc}
\hline $\begin{array}{l}\text { Aspectos de la actuación } \\
\text { gubernamental evaluados } \\
\text { por la ciudadanía }\end{array}$ & $\begin{array}{c}\text { Valoración media del aspecto } \\
\text { de los ciudadanos que } \\
\text { señalan que el principal } \\
\text { beneficiado es el gobierno }\end{array}$ & $\begin{array}{c}\text { Valoración media del aspecto } \\
\text { de los ciudadanos que } \\
\text { señalan que el principal } \\
\text { beneficiado son otros } \\
\text { actores distintos al gobierno }\end{array}$ & \begin{tabular}{c} 
Sig. \\
\hline Información características de la obra
\end{tabular} \\
\hline Información cierres vialidades & 4,46 & 5,19 & 0,010 \\
\hline Información apoyos a afectados & 4,43 & 5,17 & 0,004 \\
\hline Posibilidad presentar quejas & 2,65 & 3,13 & 0,049 \\
\hline
\end{tabular}

Fuente: elaboración propia.

Indicar que el principal beneficiario de la L3TL va a ser el gobierno implica en todos los aspectos revisados otorgar una menor calificación a la actuación del gobierno. La mayor distancia en la diferencia de calificaciones se da para el caso de la facilidad para presentar quejas y denuncias. Aquí, pensar que el principal beneficiario de la L3TL no va a ser el gobierno incrementa la valoración dada en un 33\% respecto a quienes piensan que el principal beneficiario va a ser este actor. En el resto de aspectos las diferencias de medias van del 18\% (en el caso de la información de los apoyos a afectados), al 16\% (en el caso de la información sobre las características de la obra).

Este menor peso de la variable sobre la opinión de quién es el principal beneficiario se refleja igualmente en que sólo entra en el modelo explicativo de la correlación logística binaria en el caso de la valoración sobre la actuación del gobierno a la hora de favorecer la presentación de quejas y reclamaciones. En este caso, el señalar que el principal beneficiario de la línea no va a ser el gobierno, multiplica por 1,510 las opciones de aprobar la actuación del gobierno a la hora de posibilitar la presentación de quejas y reclamaciones (sig. $0,037)$.

\subsection{La opinión sobre la influencia de la obra en la colonia}

Teóricamente se señaló que la percepción de los ciudadanos sobre cómo la infraestructura iba a modificar el día a día en su colonia o vecindario era uno de los aspectos más importantes que influían en la valoración de la actuación de los gobiernos. Para medir la percepción sobre cómo está influyendo en el presente la construcción se generó un índice que integra la valoración de los siguientes indicadores: 1) La seguridad ciudadana; 2) La existencia de parques y áreas verdes; 3) La facilidad para caminar por la colonia; 4) la limpieza e imagen de la colonia; 5) la presencia de vecinos en las calles y la animación del barrio. Los valores que puede tomar el índice son: la construcción ha mejorado, no ha incidido, o ha empeorado la calidad de vida en la colonia. Lo mismo se hizo para valorar la percepción sobre la influencia en el futuro: se construyó un índice con los indicadores mencionados, que precisa si la L3TL mejorará, no influirá, o empeorará la calidad de vida en la colonia.

La percepción sobre cómo la construcción de la L3TL está modificando la calidad de vida de la colonia es muy determinante. Las diferencias de media encontradas son todas significativas y se resumen en Tabla 7. 
GAPP. Nueva Época - N. 21, Mayo-Octubre 2019 - ISSN: 1989-8991 - DOI: 10.24965/gapp.v0i21.10537 - [Págs. 109-124]

¿Qué factores aumentan la aprobación al gobierno en un proyecto de infraestructura urbana? La construcción de la Línea 3 del Tren..

Fernando Calonge Reillo

TABLA 7. DIFERENCIAS DE MEDIAS EN LAS CALIFICACIONES DE LOS CIUDADANOS A LA ACTUACIÓN GUBERNAMENTAL, SEGÚN OPINIÓN SOBRE CÓMO INCIDE LA CONSTRUCCIÓN DE LA L3TL EN LA CALIDAD DE VIDA DE LA COLONIA

\begin{tabular}{lcccc}
\hline $\begin{array}{l}\text { Aspectos de la actuación } \\
\text { gubernamental evaluados } \\
\text { por la ciudadanía }\end{array}$ & $\begin{array}{l}\text { Valoración media } \\
\text { de los ciudadanos } \\
\text { que piensan que la } \\
\text { obra ha mejorado la } \\
\text { calidad de la colonia }\end{array}$ & $\begin{array}{l}\text { Valoración media de } \\
\text { los ciudadanos que } \\
\text { piensan que la obra } \\
\text { no ha influido en la } \\
\text { calidad de la colonia }\end{array}$ & $\begin{array}{c}\text { Valoración media de } \\
\text { los ciudadanos que } \\
\text { piensan que la obra } \\
\text { ha empeorado la } \\
\text { calidad de la colonia }\end{array}$ & Sig. \\
\hline $\begin{array}{l}\text { Información características } \\
\text { de la obra }\end{array}$ & 6,25 & 5,28 & 4,16 & 0,000 \\
\hline $\begin{array}{l}\text { Información cierres } \\
\text { vialidades }\end{array}$ & 5,75 & 5,27 & 4,19 & 0,000 \\
\hline $\begin{array}{l}\text { Información impactos } \\
\text { ambientales colonia }\end{array}$ & 4,80 & 4,36 & 3,18 & 0,000 \\
\hline $\begin{array}{l}\text { Información apoyos a } \\
\text { afectados }\end{array}$ & 3,30 & 3,24 & 2,38 \\
\hline \begin{tabular}{l} 
Posibilidad presentar quejas \\
\hline
\end{tabular} & 3,40 & 3,17 & 2,09 & 0,001 \\
\hline
\end{tabular}

Fuente: elaboración propia.

La diferencia de medias más abultada se presenta a la hora de valorar la posibilidad de presentar quejas. Aquí, el pensar que la construcción de la L3TL ha mejorado la calidad de vida en la colonia implica otorgar una calificación un $63 \%$ mayor a la actuación del gobierno, frente a quienes piensan que la construcción ha empeorado la calidad de vida. Diferencias de media igualmente sustantivas (del $51 \%$ y $50 \%$ ) se dan en la valoración de la información de impactos ambientales y en la valoración sobre las características de la obra entre quienes piensan que la obra ha mejorado la calidad de vida de la colonia frente a quienes piensan que la calidad de vida ha empeorado por la construcción de la obra.

Dada la importancia que tiene este tipo de apreciaciones de la influencia de la construcción en la calidad de vida de la colonia, esta variable ha entrado en los modelos explicativos en dos de los cinco aspectos como se valora la actuación del gobierno, como se comprueba en la Tabla 8.

TABLA 8. INCREMENTO DE OPCIONES DE APROBAR LA ACTUACIÓN DEL GOBIERNO SEGÚN SE SEÑALA QUE LA CONSTRUCCIÓN DE LA L3TL HA MEJORADO LA CALIDAD DE VIDA EN LA COLONIA

\begin{tabular}{|c|c|c|}
\hline $\begin{array}{l}\text { Aspectos de la actuación } \\
\text { gubernamental }\end{array}$ & $\begin{array}{l}\text { Incremento de opciones de aprobar esos aspectos de } \\
\text { quienes perciben mejora en la calidad de vida de la colonia }\end{array}$ & Sig. \\
\hline Información características de la obra & 4,767 & 0,011 \\
\hline Posibilidad presentar quejas & 3,396 & 0,017 \\
\hline
\end{tabular}

Fuente: elaboración propia.

Como se aprecia, indicar que la calidad de vida en la colonia ha mejorado con la construcción de la L3TL implica multiplicar por 4,767 las opciones de aprobar la actuación del gobierno en la información sobre las características de la obra respecto a quienes señalan que la calidad de vida ha empeorado. En otro de los aspectos donde se aprecia también una gran influencia es en la posibilidad de presentar quejas. En esta materia, quienes señalan que la calidad de vida de la colonia ha mejorado con la construcción de la L3TL multiplican por 3,396 sus opciones de aprobar la actuación del gobierno.

La perspectiva a futuro sobre cómo incidirá la L3TL en la calidad de vida de la colonia en la valoración de la actuación gubernamental también es apreciable, aunque menor que en el anterior caso sobre la valoración de la incidencia presente de la obra. Las diferencias de medias en todos los casos son significativas estadísticamente, según se aprecia en la Tabla 9. 
GAPP. Nueva Época - N. 21, Mayo-Octubre 2019 - ISSN: 1989-8991 - DOI: 10.24965/gapp.v0i21.10537 - [Págs. 109-124] ¿Qué factores aumentan la aprobación al gobierno en un proyecto de infraestructura urbana? La construcción de la Línea 3 del Tren..

Fernando Calonge Reillo

TABLA 9. DIFERENCIAS DE MEDIAS EN LAS CALIFICACIONES DE LOS CIUDADANOS A LA ACTUACIÓN GUBERNAMENTAL, SEGÚN OPINIÓN SOBRE CÓMO INCIDIRÁ LA L3TL EN LA CALIDAD DE VIDA DE LA COLONIA

\begin{tabular}{|c|c|c|c|c|}
\hline $\begin{array}{l}\text { Aspectos de la actuación } \\
\text { gubernamental evaluados } \\
\text { por la ciudadanía }\end{array}$ & $\begin{array}{l}\text { Valoración media de } \\
\text { los ciudadanos que } \\
\text { piensan que la obra } \\
\text { mejorará la calidad } \\
\text { de la colonia }\end{array}$ & $\begin{array}{c}\text { Valoración media de } \\
\text { los ciudadanos que } \\
\text { piensan que la obra } \\
\text { no influirá en la } \\
\text { calidad de la colonia }\end{array}$ & $\begin{array}{l}\text { Valoración media } \\
\text { de los ciudadanos } \\
\text { que piensan que la } \\
\text { obra empeorará la } \\
\text { calidad de la colonia }\end{array}$ & Sig. \\
\hline $\begin{array}{l}\text { Información características } \\
\text { de la obra }\end{array}$ & 4,43 & 5,51 & 3,61 & 0,000 \\
\hline Información cierres vialidades & 4,68 & 5,34 & 3,83 & 0,000 \\
\hline $\begin{array}{l}\text { Información impactos } \\
\text { ambientales colonia }\end{array}$ & 3,44 & 4,51 & 3,17 & 0,000 \\
\hline Información apoyos a afectados & 2,61 & 3,33 & 2,26 & 0,000 \\
\hline Posibilidad presentar quejas & 2,36 & 3,27 & 2,17 & 0,000 \\
\hline
\end{tabular}

Fuente: elaboración propia.

El principal dato que llama más la atención, y que merecerá una posterior investigación, es que quienes afirman que la calidad de vida en la colonia seguirá igual con la L3TL, dan siempre una mejor calificación en todos los aspectos considerados sobre la actuación del gobierno, que quienes señalan que la calidad de vida en la colonia mejorará. La lectura de estos datos no es fácil, por lo que deben explorarse a futuro con mayor detenimiento. Dejada de lado esta anomalía, sigue reproduciéndose la situación de que quienes afirman que mejorará la calidad de vida en todos los aspectos dan una mejor calificación a la actuación del gobierno que quienes señalan que empeorará.

La influencia de esta anomalía de que la posición intermedia en la valoración a futuro de la colonia será de «igual» mejora la valoración de la actuación de los distintos niveles de gobierno, y la menor diferencia de medias entre los distintos valores sobre la percepción a futuro de la colonia hace que esta variable no haya entrado en ningún modelo explicativo sobre cómo aumentan las probabilidades de aprobar la actuación gubernamental.

\subsection{La participación en la determinación de las infraestructuras urbanas}

Teóricamente se ha advertido cómo el nivel de participación de la población puede ser determinante del grado de aprobación que recibe la actuación del gobierno en la construcción de infraestructuras y grandes proyectos, aunque no se ha terminado de alcanzar un consenso sobre la dirección y la forma de esa relación. En la presente investigación también se ha podido constatar la existencia de esta relación.

El cuestionario empleado introdujo la pregunta sobre si el ciudadano había participado en asambleas o reuniones de vecinos, convocadas con relación a la construcción de la L3TL. Cuando vinculamos las respuestas a estas preguntas con las variables sobre la actuación del gobierno, encontramos que las diferencias de medias son significativas, excepto para el aspecto que valora cómo el gobierno ha aportado información sobre los impactos ambientales en la colonia. Los resultados se recogen en la Tabla 10.

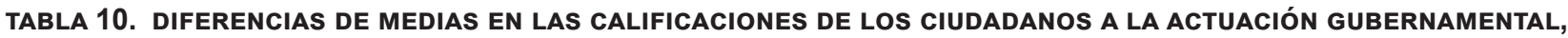
SEGÚN PARTICIPACIÓN EN ASAMBLEAS O REUNIONES DE VECINOS

\begin{tabular}{lccc}
\hline $\begin{array}{l}\text { Aspectos de la actuación gubernamental } \\
\text { evaluados por la ciudadanía }\end{array}$ & $\begin{array}{c}\text { Evaluación media } \\
\text { de los ciudadanos } \\
\text { que han participado }\end{array}$ & $\begin{array}{c}\text { Evaluación media } \\
\text { de los ciudadanos } \\
\text { que no han participado }\end{array}$ & \begin{tabular}{c} 
Sig. \\
\hline Información características de la obra
\end{tabular} \\
\hline Información cierres vialidades & 7,31 & 4,94 & 0,000 \\
\hline Información apoyos a afectados & 6,34 & 4,95 & 0,008 \\
\hline Posibilidad presentar quejas & 4,79 & 2,96 & 0,001 \\
\hline
\end{tabular}

Fuente: elaboración propia. 
Los instrumentos de participación implementados alrededor de la construcción de la L3TL han sido muy escasos. Sólo el $3,71 \%$ de los ciudadanos encuestados habían participado en alguna asamblea o reunión relacionada con esta obra. Sin embargo se observa que incrementar estos mecanismos de participación habría podido incrementar la aceptación general sobre la actuación del gobierno, dado que siempre quienes participaron dan una calificación muy superior. Esto es especialmente cierto en la evaluación de la información dada sobre los apoyos a los afectados, y ante la posibilidad de presentar quejas y denuncias. En el primer caso la calificación media de los que participaron fue un $62 \%$ superior, y en el segundo fue un $61 \%$ superior respecto a quienes no participaron.

De igual manera, como se aprecia en la Tabla 11, la participación ciudadana ha sido significativa y ha quedado integrada en el modelo en la explicación de tres de los cinco aspectos de la actuación del gobierno sometidos a juicio.

TABLA 11. INCREMENTO DE OPCIONES DE APROBAR LA ACTUACIÓN DEL GOBIERNO SEGÚN SE HA PARTICIPADO EN REUNIONES O ASAMBLEAS DE VECIONS

\begin{tabular}{lcc}
\hline Aspectos de la actuación gubernamental & $\begin{array}{c}\text { Incremento de opciones de aprobar esos } \\
\text { aspectos de quienes han participado }\end{array}$ & Sig. \\
\hline Información cierres vialidades & 4,291 & 0,015 \\
\hline Información apoyo afectados & 6,536 & 0,000 \\
\hline Posibilidad presentar quejas & 5,735 & 0,000 \\
\hline
\end{tabular}

Fuente: elaboración propia.

Así, los resultados de la regresión logística binaria indican que haber participado incrementa 6,536 veces las opciones de aprobar la actuación del gobierno en materia de dar información sobre apoyos a los afectados; incrementa 5,735 veces las opciones de aprobar esa actuación en la facilidad de recibir quejas y denuncias; y multiplica 4,291 las opciones de aprobar la actuación del gobierno sobre la información dada ante los cierres de vialidades.

\section{CONCLUSIONES}

La construcción de grandes proyectos y de infraestructuras urbanas es un fenómeno que suscita mucho interés y atención públicos por el elevado número de repercusiones que acarrea. Desde este punto de vista el debate ciudadano tiende a polarizarse en la forma como se valora el desempeño gubernamental. En este artículo he intentado mostrar algunos condicionantes de la diferente valoración que dan los ciudadanos a esa acción gubernamental, en el caso de la construcción de la L3TL en el Área Metropolitana de Guadalajara, México.

El presente artículo ha mostrado que los factores que de manera más determinante influían en la evaluación de la acción gubernamental estaban relacionados con la participación ciudadana. A pesar de las escasas y muy escuetas fórmulas de participación ideadas para el caso de la L3TL, según la tradicional fórmula de gestionar la infraestructura en la región, los vecinos que participaban en asambleas y reuniones convocadas al efecto mostraron una posición mucho más favorable sobre la acción del gobierno que quienes no participaban.

Junto a la participación, el señalamiento de que no se advertían impactos negativos en la obra derivados de la construcción, también influyó positivamente en la valoración de la acción gubernamental en todos los aspectos analizados. Es más, en las ocasiones en que los vecinos indicaban que la construcción había mejorado la calidad de vida de la colonia el apoyo y la aprobación de la acción gubernamental se multiplicaba varias veces respecto a quienes señalaban que la obra había empeorado esa misma calidad de vida.

Asimismo, aspectos más vagos y relacionados con la influencia de la obra en la mejora futura de la calidad de vida de la colonia, su necesidad y quién iba a ser el principal beneficiado, también influyeron en la evaluación de la actividad del gobierno, pero de una forma más vaga.

En el señalado contexto de la región latinoamericana, donde se ha consignado que está más presente un modelo tecnocrático y autoritario en la implementación de las infrestructuras, mejorar los mecanismos 
de gobernanza urbana en estos dos rubros puede ser especialmente importante para mejorar los niveles de aceptación de la labor gubernamental. Los resultados de la investigación muestran que una mayor atención por evitar los impactos de la construcción de la infraestructura sobre la ciudadanía, y que una ampliación de los mecanismos de participación mejora la evaluación que los ciudadanos realizan sobre la actuación de los diferentes niveles de gobierno.

La investigación en que se fundamentan estos resultados fue realizada durante el período de construcción de la infraestructura, por lo que es posible que las valoraciones generales de la infraestructura estén influidas desde los impactos negativos sufridos durante el mismo. Por ello, sería recomendable realizar futuras mediciones en períodos de operación de la infraestructura, para calibrar si el posible disfrute de sus beneficios altera de alguna manera la aprobación de la actuación de los distintos niveles de gobierno.

Futuras investigaciones habrán de profundizar también en el conocimiento de cómo determinadas variables de índole socioeconómica de los sujetos pueden estar incidiendo en los niveles de aceptación de la actuación gubernamental. Aunque se tuvo la precaución de incluir variables como el nivel de estudios o la clase social auto-percibida, éstas no resultaron significativas a la hora de intentar predecir la variable dependiente, algo que puede ser imputado a un escaso tamaño muestral. Futuras investigaciones habrán de tener cuidado de mejorar el diseño metodológico para poder probar más sólidamente la influencia de este tipo de variables.

Finalmente, será necesario profundizar en los resultados obtenidos por este trabajo haciendo una indagación sobre cómo aspectos relacionados con la cultura política de los ciudadanos inciden en su aprobación de la actuación del gobierno en la implementación de infraestructura urbana. Próximas investigaciones cuantitativas y cualitativas podrán indagar cómo el recuerdo de voto partidista fue importante para deparar variaciones en la evaluación de los partidos políticos al frente de los gobiernos encargados de la construcción de la infraestructura, pero también podrán demostrar cómo determinadas formas y culturas políticas detonan patrones diferenciados de aceptación.

\section{REFERENCIAS}

ALLEN, J. y LLOYD JONES, T. (2010): "Neighborhood Asset Management: Life Cycles and Learning for Social Sustainability", en MANZI, T. et al. (eds.): Social Sustainability in Urban Areas. Communities, Connectivity and the Urban Fabric, págs. 65-82. London: Earthscan.

ANAND, N. (2011): "Pressure: The Poli-Technics of Water Supply in Mumbay", en Cultural Anthropology, vol. 26, núm. 4, págs. 542-564. DOI: https://doi.org/10.1111/j.1548-1360.2011.01111.x.

BAKKER, K. (2010): Privatizing water. Governance failure and the world's urban water crisis. London: Cornell University Press.

BARKER, J. (2005): "Engineers and Political Dreams: Indonesia in the Satellite Age", en Current Anthropology, vol. 46, núm. 5, págs. 703-727. DOI: https://doi.org/10.1086/432652.

BATTAGLIA, M., MELONI, E. y CAUTILLO, A. (2014): "Technical Assesments and Public Perception of Environmental Issues. The Case of the Municipality of Pisa", en Local Environment, vol. 19, núm. 7, págs. 786-802. DOI: https:// doi.org/10.1080/13549839.2013.792049.

BON, B. (2015): "A New Megaproject Model and a New Funding Model. Travelling Concepts and Local Adaptations around the Delhi Metro", en Habitat International, vol. 45, págs. 223-230. DOI: https://doi.org/10.1016/j. habitatint.2014.06.008.

BRODZINSKY, S. (2014): “A Tale of Two Cities. Bogota”, en Americas Quaterly, Winter 2014, págs. 74-82.

BUNNELL, T. (2002): "Kampung Rules. Landscape and the Contested Government of Urban(e) Malayness", en Urban Studies, vol. 39, núm. 9, págs. 1.685-1.701. DOI: https://doi.org/10.1080/00420980220151727.

DA SILVA, A. (2010): "Sao Paulo and the Challenges for Social Sustainability: The Case of an Urban Housing Policy", en POLESE, M. y STREN, R. (eds.): The Social Sustainability of Cities: Diversity and the Management of Change, págs. 202-227. Toronto: University of Toronto Press.

DALAKOGLOU, D. (2010): "The Road: An Ethnography of the Albanian-Greek Cross Border Motorway", en American Anthropologist, vol. 37, núm. 1, págs. 132-149. DOI: https://doi.org/10.1111/j.1548-1425.2010.01246.x.

DÁVILA, J. y BRAND, P. (2010): "La gobernanza del transporte público urbano. Indagaciones alrededor de los metrocables de Medellín", en Revista Bitácora Urbano Territorial, vol. 21, núm. 2, págs. 85-96.

DIETZ, S. y ATKINSON, G. (2005): "Public Perceptions of Equity in Environmental Policy. Traffic Emissions Policy in an English Urban Area”, en Local Environment, vol. 10, núm. 4, págs. 445-459. DOI: https://doi. org/10.1080/13549830500160982.

DUPONT, V. D. N. (2011): "The Dream of Delhi as a Global City", en International Journal of Urban and Regional Research, vol. 35, núm. 3, págs. 533-554. DOI: https://doi.org/10.1111/j.1468-2427.2010.01027.x. 
EVANS, P. (2002): "Introduction. Looking for Agents of Urban Livability in a Globalized Political Economy", en EVANS, P. (ed.): Livable Cities? Urban Struggles for Livelihood and Sustainability, págs. 1-30. Berkeley: University of California Press.

FLYVBJERG, B. (2005): "Machiavellian Megaprojects", en Antipode, vol. 37, núm. 1, págs. 18-22. DOI: https://doi. org/10.1111/j.0066-4812.2005.00471.x.

FLYVBJERG, B. (2009): "Survival of the unfittest: why the worst infrastructure gets built - and what we can do about it", en Oxford Review of Economic Policy, vol. 25, núm. 3, págs. 344-367. DOI: https://doi.org/10.2139/ssrn.2229768.

FLYVBJERG, B. (2014): "What You Should Know About Megaprojects and Why: An Overview", en Project Management Journal, vol. 45, núm. 2, págs. 6-19. DOI: https://doi.org/10.1002/pmj.21409.

GRIECO, M. (2015): "Social Sustainability and Urban Mobility. Shifting to a Socially Responsible Pro-Poor Perspective", en Social Responsibility Journal, vol. 11, núm. 1, págs. 82-97. DOI: https://doi.org/10.1108/srj-05-2014-0061.

GROVE, K. (2009): "Rethinking the Nature of Urban Environmental Politics: Security, Subjectivity and the Non-Human", en Geoforum, núm. 40, págs. 207-216. DOI: https://doi.org/10.1016/j.geoforum.2008.09.005.

HUMPHREY, C. (2003): "Ideology in Infrastructure. Architecture and Soviet Imagination", en Journal of the Royal Anthropological Institute, núm. 11, págs. 39-58. DOI: https://doi.org/10.1111/j.1467-9655.2005.00225.x.

HARVEY, P. (2012): "The Topological Quality of Infrastructural Relation. An Ethnographic Approach”, en Theory, Culture and Society, vol. 29, núm. 4-5, págs. 76-92. DOI: https://doi.org/10.1177/0263276412448827.

HESS, D. J. (2009): Localist Movements in a Global Economy. Sustainability, Justice and Urban Development in the United States. London: The MIT Press.

JANSE, G. y KONIJNENDIJK, C. C. (2007): "Communication Between Science, Policy and Citizens in Public Participation in Urban Forestry. Experiences from Neighborwoods Project", en Urban Forestry and Urban Greening, núm. 6, págs. 23-40. DOI: https://doi.org/10.1016/j.ufug.2006.09.005.

JORDHUS LIER, D. (2015): "Community resistance against megaprojects: The case of the N2 Gateway project in Joe Slovo informal settlement, Cape Town”, en Habitat International, vol. 45, págs. 169-176. DOI: https://doi. org/10.1016/j.habitatint.2014.02.006.

KAIKA, M. y SWYNGEDOUW, E. (2000): "Fetishizing the Modern City: The Phantasmagoria or Urban Technological Networks", en International Journal of Urban and Regional Research, vol. 24, núm. 1, págs. 120-138. DOI: https:// doi.org/10.1111/1468-2427.00239.

KASH, G. e HIDALGO, D. (2014): "The promise and challenges of integrating public transportation in Bogotá, Colombia”, en Public Transport, vol. 6, págs. 107-135. DOI: https://doi.org/10.1007/s12469-013-0083-7.

KECK, M. E. (2002): "Water, Water, Everywhere, Nor Any Drop to Drink. Land Use and Water Policy in Sao Paulo, Brazil", en EVANS, P. (ed.): Livable Cities? Urban Struggles for Livelihood and Sustainability, págs. 162-194. Berkeley: University of California Press.

KHAN, N. (2006): "Flaws in the Flow. Roads and their Modernity in Pakistan", en Social Text, vol. 24, núm. 4, págs. 87113. DOI: $h$ ttps://doi.org/10.1215/01642472-2006-012.

LARKIN, B. (2013): "The Politics and Poetics of Infrastructure”, en Annual Review of Anthropology, vol. 42, págs. 327343. DOI: $h$ ttps://doi.org/10.1146/annurev-anthro-092412-155522.

LARKING, B. (2008): Signal and Noise. Media, Infrastructure and Urban Culture in Nigeria. London: Duke University Press.

LEVINE, M. V. (2000): "A Third World City in the First World? Social Exclusion, Racial Inequality and Sustainable Development in Baltimore, Maryland", en POLESE, M. y STREN, R. (eds.): The Social Sustainability of Cities. Diversity and the Management of Change, págs. 122-156. London: University of Toronto Press.

MCCRIGHT, A. y CLARK, T. N. (2006): "The political opportunity structure of the environmental movement in the U. S. communities”, en MCCRIGHT, A. y CLARK, T. N. (eds.): Community and Ecology: Dynamics of Place, Sustainability, and Politics, págs. 199-240. New York: Elsevier.

MANZI, T. (2010): "Creating Sustainable Neighborhoods? The Development and Management of Mixed Income Communities”, en MANZI, T. et al. (eds.): Social Sustainability in Urban Areas. Communities, Connectivity and the Urban Fabric, págs. 35-48. London: Earthscan.

MANZI, T., LUCAS, K., LLOYD JONES, T. y ALLEN, J. (2010): “Understanding Social Sustainability: Key Concepts and Developments in Theory and Practice", en MANZI, T. et al. (eds.): Social Sustainability in Urban Areas. Communities, Connectivity and the Urban Fabric, págs. 1-34. London: Earthscan.

MARINCIONI, F. y APPIOTI, F. (2009): "The Lyon-Turin High Speed Rail. The Public Debate and Perception of Environmental Risk in Susa Valley, Italy”, en Environmental Management, vol. 43, núm. 5, págs. 863-875. DOI: https://doi.org/10.1007/s00267-009-9271-2.

MATTELART, A. y MATTELART, M. (1997): Historia de las Teorías de la Comunicación. Barcelona: Paidós.

MORENO CARRANCO, M. (2013): "Global Mexico under Construction. The Santa Fe Megaproject in Mexico City", en IRAZABAL, C. (ed.): Transbordering Latin Americas. Liminal Places, Cultures and Powers, págs. 187-214. New York: Routledge.

NORTON, B. (2002): "Ecology and Opportunity: Intergenerational Equity and Sustainable Options", en DOBSON, A. (ed.): Fairness and Futurity. Essays on Environmental Sustainability and Social Justice, págs. 118-150. Oxford: Oxford University Press. 
NOVY, J. y PETERS, D. (2013): "Railways Megaprojects as Catalysts for the Remaking of Post-Industrial Cities? The Case of Stuttgart 21 in Germany", en DEL CERRO SANTAMARÍA, G. (ed.): Urban Megaprojects. A Worldwide Review, págs. 237-262. Bingley: Emerald.

PAGET-SEEKINS, L. (2015): "Bus Rapid Transit as a Neoliberal Contradiction", en Journal of Transport Geography, vol. 48, págs. 115-120. DOI: https://doi.org/10.1016/j.jtrangeo.2015.08.015.

PÉREZ NEGRETE, M. (2016): "Ciudad de México: el camino recorrido en la conformación de una ciudad global”, en Revista Mexicana de Ciencias Políticas y Sociales, núm. 226, págs. 331-352. DOI: https://doi.org/10.1016/s01851918(16)30013-7.

RUMING, K. J. (2014): "Urban Consolidation, Strategic Planning and Community Opposition in Sydney, Australia. Unpacking Policy Knowledge and Public Perceptions”, en Land Use Policy, vol. 39, págs. 254-265. DOI: https:// doi.org/10.1016/j.landusepol.2014.02.010.

SACK, D. (2011): "Governance failures in integrated transport policy. On mismatch of «co-opetition" in multi level analysis”, en German Policy Studies, vol. 7, núm. 2, págs. 43-70.

SCHNEIER-MADANES, G. (2005): "Conflicts and the Rise of Users' Participation in Buenos Aires Water Supply Concession, 1993-2003”, en COUTARD, O., HANLEY, R. E. y ZIMMERMAN, R. (eds.): Sustaining Urban Networks. The Social Diffusion of Large Technical Systems, págs. 151-171. London: Routledge.

SCHOT, J. (2003): "The Contested Rise of a Modernist Technology Politics", en MISA, T. J., BREY, P. y FEENBERG, A. (eds.): Modernity and Technology, págs. 257-302. London: The MIT Press.

SIEMIATYCKI, M. (2006): "Message in a Metro. Building Urban Rail Infrastructure and Image in Delhi, India", en International Journal of Urban and Regional Research, vol. 30, núm. 2, págs. 277-292. DOI: https://doi.org/10.1111/ j.1468-2427.2006.00664.x.

SIEMIATYCKI, M. (2005): "Beyond Moving People: Excavating the Motivations for Investing in Urban Public Transit Infrastructure in Bilbao, Spain", en European Planning Studies, vol. 13, núm. 1, págs. 23-44. DOI: https://doi.org/1 $0.1080 / 0965431042000312398$.

SMITH, A. (2010): "The Relations Between Major Events, the Urban Fabric and Social Sustainability”, en MANZI, T. et al. (eds.): Social Sustainability in Urban Areas. Communities, Connectivity and the Urban Fabric, págs. $199-220$. London: Earthscan.

STREN, R. y POLESE, M. (2000): "Understanding the New Sociocultural Dynamics of Cities: Comparative Urban Policy in Global Context", en POLESE, M. y STREN, R. (eds.): The Social Sustainability of Cities: Diversity and the Management of Change, págs. 3-38. Toronto: University of Toronto Press.

TURNER, J. (2012): "Urban Mass Transit, Gender Planning Protocols and Social Sustainability. The Case of Jakarta", en Research in Transportation Economics, núm. 34, págs. 48-53. DOI: https://doi.org/10.1016/j.retrec.2011.12.003.

VALENTIN, V. y BOGUS, S. M. (2013): "Public Opinion as an Indicator of Social Sustainability of Construction Projects", en International Conference on Sustainable Design, Engineering and Construction, año 2012, págs. 561-568.

VALENZUELA, A. (2013): "Dispositivos de la globalización: la construcción de grandes proyectos en la Ciudad de México", en EURE, vol. 38, núm. 116, págs. 101-118. DOI: https://doi.org/10.4067/s0250-71612013000100004. 